\title{
Multi-Analyte Fluorescent Detection
}

National Cancer Institute

\section{Source}

National Cancer Institute. Multi-Analyte Fluorescent Detection. NCI Thesaurus. Code C92628.

A method for simultaneous detection of multiple target molecules. It utilizes specific antibodies for the analytes of interest coupled to fluorescent polystyrol beads. After conjug ation of the captured analyte to the bead, the beads are differentiated by their size and their spectral signature, allowing quantitation of analyte concentration in the sample. 